\title{
Propagation automatique des incertitudes : application aux techniques d'auto-étalonnage des analyseurs de réseau vectoriels
}

\author{
Djamel Allal ${ }^{1 a}$, Blair Hall ${ }^{2}$, Patricia Vincent ${ }^{1}$, Alexis Litwin ${ }^{1}$ et François Ziadé ${ }^{1}$ \\ ${ }^{1}$ Laboratoire National de Métrologie et d'Essais LNE, France \\ ${ }^{2}$ Measurement Standards Laboratory of New Zealand, Callaghan Innovation, New Zealand.
}

\begin{abstract}
Some important vector network analyser (VNA) calibration procedures, such as thru-reflect-line (TRL), are not amenable to propagation of measurement uncertainty by conventional methods, because the equations involved do not explicitly refer to estimates of calibration standards used. However, the sensitivity of calibration constants to those estimates can be found by relating changes in standard values to changes in VNA readings. This is easily implemented with new algorithmic methods of uncertainty propagation.
\end{abstract}

\section{Introduction}

Certaines procédures d'étalonnage de l'analyseur de réseau vectoriel (VNA, pour Vector Network Analyser en anglais) comme la TRL (Thru-Reflect-Line) [1], ne permettent pas la propagation des incertitudes par les méthodes conventionnelles. Les équations d'étalonnage utilisées pour estimer les paramètres du modèle ne faisant pas référence aux valeurs des étalons utilisés, les dérivées partielles nécessaires pour la propagation des incertitudes ne peuvent ainsi pas être évaluées directement. Cependant, les coefficients de sensibilité des paramètres du modèle peuvent être déterminés de manière indirecte en se référant aux variations dans la réponse du VNA dues à des petites variations de la valeur des étalons. Nous décrivons cette approche et nous montrons qu'elle peut être implémentée avec les nouvelles méthodes algorithmiques de propagation des incertitudes.

$\mathrm{Au}$ paragraphe 2, nous expliquons pourquoi les incertitudes dues aux étalons ne peuvent pas être propagées de manière classique dans le cas de méthodes d'étalonnage telles que la TRL. Le paragraphe 3 décrit une méthode indirecte permettant d'obtenir les sensibilités des paramètres estimés, aux valeurs attribuées aux étalons (valeurs étalons), en évaluant d'abord la sensibilité des observations brutes aux écarts par rapport aux valeurs étalons idéales et ensuite en les propageant via les équations d'étalonnage. Au paragraphe 4, nous montrons comment cette méthode peut être appliquée au cas de l'étalonnage TRL. Enfin, au paragraphe 5 nous appliquons ce procédé à un étalonnage TRL effectué à l'aide d'un kit d'étalonnage sur connecteur PC7 entre $2 \mathrm{GHz}$ et $18 \mathrm{GHz}$ suivi de la mesure d'un atténuateur de $20 \mathrm{~dB}$.

\footnotetext{
a Djamel Allal : djamel.allal@Ine.fr
}

\section{Procédure d'(auto-)étalonnage TRL}

Parmi les instruments de mesure modernes, le VNA ne peut être utilisé sans corriger ses erreurs systématiques. Cette correction nécessite de mesurer au préalable un jeu d'étalons (plusieurs étalons sont nécessaires). Plusieurs modèles et méthodes ont été développés pour estimer les erreurs du VNA.

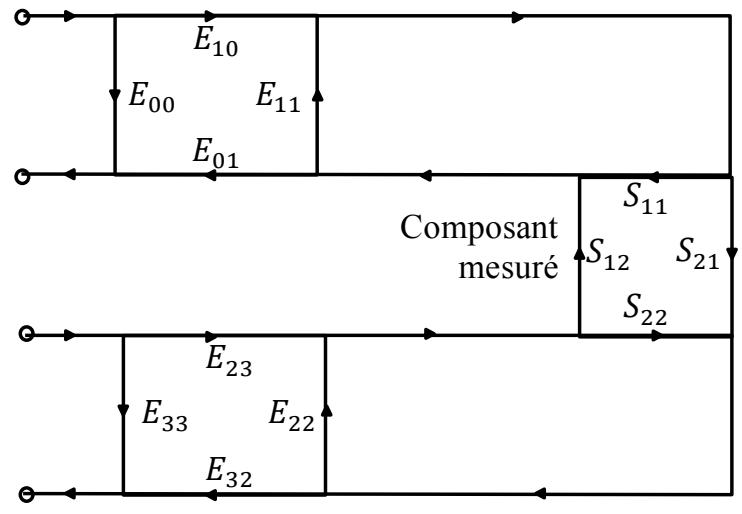

Figure 1. Modèle du VNA à 8 termes d'erreur (7 par normalisation)

Parmi les méthodes, la TRL est considérée comme une procédure fondamentale pour les mesures de précision $[2,3,4]$. La TRL permet d'estimer les paramètres d'un modèle à 8 termes d'erreur (figure 1), ou sept termes d'erreur après normalisation, en utilisant la mesure d'une ligne de transmission (Line), la mesure de la connexion directe des deux accès du VNA (Thru) et la mesure aux deux accès du VNA de deux charges identiques, à forte réflexion (Reflect). Les équations de la 
TRL peuvent être établies sous différentes formes, mais dans tous les cas, elles ne réfèrent qu'aux mesures brutes et pas aux valeurs étalons.

En effet, la méthode TRL, comme d'autres méthodes utilisant le modèle à 7 termes d'erreur (TRM, LRM, TAN, etc.) prennent comme impédance de référence, l'impédance d'un ou plusieurs étalons du kit d'étalonnage. Par exemple, pour la TRL, l'impédance de référence est l'impédance caractéristique de la ligne de transmission. Ainsi référencée, la matrice $\mathrm{S}$ de la ligne de transmission s'écrit :

$$
\mathbf{S}=\left(\begin{array}{ll}
0 & T \\
T & 0
\end{array}\right)
$$

où $T=\exp (-\gamma l)$ est le facteur de transmission de la ligne, fonction de la constante de propagation $\gamma$ et de la longueur $l$ de la ligne. $T$ n'est pas vu comme une valeur étalon, mais comme une inconnue du système d'équations et est déterminé pendant l'exécution de l'algorithme TRL (auto-étalonnage). Quant aux valeurs étalons c'est-à-dire les paramètres $S_{11}$ et $S_{22}$, elles sont nulles et n'apparaissent pas dans les équations d'étalonnage.

Ceci pose un problème pour l'évaluation des incertitudes de mesure qui dépendent, parmi toutes les grandeurs d'influence, de la sensibilité du résultat aux petites variations des valeurs étalons $[5,6]$.

\section{Calcul indirect des coefficients de sensibilité}

De manière générale, une mesure brute obtenue au VNA peut être vue comme la réponse d'un instrument à un stimulus particulier, correspondant à la grandeur à mesurer. Ce modèle stimulus-réponse peut être représenté par une fonction du système de mesure

$$
\mathbf{x}_{j}=\mathbf{f}\left(\mathbf{y}_{j} ; \mathbf{e}\right)
$$

où $\mathbf{x}_{j}$ est un vecteur de réponses (paramètres $S$ bruts) à un vecteur stimulus $\mathbf{y}_{j}$ (paramètres $S$ du stimulus mesuré $j$ ) et $\mathbf{e}$ est un vecteur de paramètres représentant le modèle et généralement appelés termes d'erreur. Une procédure d'étalonnage prend un ensemble de réponses $\left\{\mathbf{x}_{j}\right\}$, correspondant à un ensemble de stimuli connus (étalons) $\left\{\mathbf{y}_{j}\right\}$, et évalue les paramètres ou termes d'erreur

$$
\mathbf{e}=\mathbf{h}\left(\left\{\mathbf{x}_{j}\right\},\left\{\mathbf{y}_{j}\right\}\right)
$$

A la suite de l'étalonnage, le modèle du système de mesure peut être inversé, pour estimer le stimulus inconnu correspondant à une réponse observée. Cela nécessite de connaître les estimées des paramètres d'erreur déterminées par l'équation (3) :

$$
\hat{\mathbf{y}}=\mathbf{g}(\mathbf{x} ; \hat{\mathbf{e}}) \equiv \mathbf{f}^{-1}(\mathbf{x} ; \hat{\mathbf{e}})
$$

Afin d'évaluer l'incertitude d'une mesure $\hat{\mathbf{y}}$, comme estimée de $\mathbf{y}$, il est nécessaire de connaître la sensibilité de la fonction $\mathbf{g}$ à chaque grandeur d'influence, y compris la sensibilité des paramètres d'erreur e à des erreurs dans les valeurs des étalons. Toutefois, la méthode d'étalonnage TRL, comme expliqué au paragraphe précédent présente une difficulté dans le sens où la fonction $\mathbf{h}$ ne se présente pas sous une forme qui peut être différentiée par rapport aux $\left\{\mathbf{y}_{j}\right\}$. Par contre, les dérivées partielles nécessaires pour la propagation des incertitudes peuvent être obtenues par une approche indirecte qui s'appuie sur l'utilisation de la fonction $\mathbf{f}$ de réponse du système, comme nous le montrons ci-dessous.

Une petite variation d'une composante d'un stimulus donné $\delta y_{i j}$ sera compensée par un ensemble donné de variations dans les réponses $\delta x_{1 j}, \delta x_{2 j}, \ldots$, laissant une composante d'erreur $e_{k}$ inchangée. En termes de différentielles nous écrivons, en considérant les éléments de $\left\{\mathbf{y}_{j}\right\}$ comme indépendants

$$
\begin{gathered}
\partial \mathrm{e}_{\mathrm{k}}=0=\frac{\partial \mathrm{e}_{\mathrm{k}}}{\partial \mathrm{x}_{1 \mathrm{j}}} \delta \mathrm{x}_{1 \mathrm{j}}+\frac{\partial \mathrm{e}_{\mathrm{k}}}{\partial \mathrm{x}_{2 \mathrm{j}}} \delta \mathrm{x}_{2 \mathrm{j}}+\cdots+\frac{\partial \mathrm{e}_{\mathrm{k}}}{\partial \mathrm{y}_{\mathrm{ij}}} \delta \mathrm{y}_{\mathrm{ij}} \\
\frac{\partial e_{k}}{\partial y_{i j}}=-\frac{\partial e_{k}}{\partial x_{1 j}} \frac{\partial x_{1 j}}{\partial y_{i j}}-\frac{\partial e_{k}}{\partial x_{2 j}} \frac{\partial x_{2 j}}{\partial y_{i j}}-\cdots
\end{gathered}
$$$$
\text { et }
$$

En d'autres termes, la sensibilité des $e_{k}$ à une variation de la i-ème composante du j-ème étalon peut être trouvée en utilisant le théorème de dérivation des fonctions composées (ou règle de la chaîne) pour combiner les sensibilités des $e_{k}$ à des variations dans les réponses, avec la sensibilité des réponses à la variation de l'étalon.

L'équation (6) permet d'obtenir les dérivées partielles nécessaires pour propager les composantes d'incertitude associées aux valeurs de l'étalon. Les autres composantes d'incertitude, associées aux mesures brutes peuvent être évaluées par ailleurs sans aucune difficulté particulière.

Ce processus peut être automatisé grâce à un logiciel utilisant un concept appelé nombre incertain qui encapsule les informations sur la valeur d'une estimée et ses sensibilités à toutes les grandeurs d'influence [7]. Lorsque des nombres incertains sont combinés dans des expressions mathématiques, la règle de la chaîne est automatiquement appliquée pour obtenir les coefficients de sensibilité du résultat et ainsi propager les incertitudes [8].

Ainsi, les étapes pour obtenir les incertitudes finales sont décrites comme suit :

- Appliquer la fonction $\mathbf{h}$ aux nombres incertains représentant les données de mesure brutes des étalons, ce qui permet d'obtenir des estimées des termes d'erreur sous forme de nombres incertains mais ne contenant que l'incertitude due aux mesures.

- En utilisant ces estimées sans leur incertitude, calculer une deuxième série de pseudo-mesures en appliquant la fonction $\mathbf{f}$ sur les nombres incertains représentant les étalons et leurs incertitudes associées.

- Appliquer à nouveau la fonction $\mathbf{h}$ à ces pseudomesures ce qui permet d'obtenir des estimées des termes 
d'erreur sous forme de nombres incertains contenant cette fois-ci les incertitudes dues aux étalons.

- Combiner les deux estimations de termes d'erreur pour obtenir les termes d'erreur finaux sous forme de nombres incertains contenant les incertitudes dues à la fois aux étalons et à la mesure.

Ainsi, même si des calculs algébriques importants sont nécessaires, il est possible de procéder à un calcul complet d'incertitude pour le cas d'un étalonnage TRL appliqué à des mesures à l'aide d'un analyseur de réseau comme démontré au paragraphe suivant.

\section{Application à la méthode TRL}

Une application du procédé décrit plus haut a déjà été démontrée dans le cas de la mesure d'une charge sur un des accès du VNA, où le modèle de mesure contient 3 termes d'erreur et la méthode généralement employée est la SOL utilisant un court-circuit, un circuit ouvert et une charge adaptée et où des valeurs idéales de facteur de réflexion de $-1,1$ et 0 ont été attribuées à ces étalons [9].

Dans la suite, nous expliquons comment ce procédé peut être appliqué à la propagation (automatique) des incertitudes dans le cas de la méthode TRL.

A partir du modèle de la figure 1, on pose

$$
\left(\begin{array}{l}
e_{1} \\
e_{2} \\
e_{3} \\
e_{4} \\
e_{5} \\
e_{6} \\
e_{7}
\end{array}\right)=\left(\begin{array}{l}
E_{00} \\
E_{11} E_{23} / E_{10} \\
\left(E_{00} E_{11}-E_{10} E_{01}\right) E_{23} / E_{10} \\
E_{22} E_{10} / E_{23} \\
E_{33} \\
\left(E_{00} E_{11}-E_{10} E_{01}\right) E_{10} / E_{23} \\
E_{10} / E_{23}
\end{array}\right)
$$

Afin de garder la notation adoptée au paragraphe précédent, pour chaque étalon mesuré, nous écrivons

$$
\left(\begin{array}{l}
x_{1} \\
x_{2} \\
x_{3} \\
x_{4}
\end{array}\right)=\left(\begin{array}{c}
{\left[\left(e_{1}-y_{1} e_{3} e_{7}\right)\left(e_{7}-y_{4} e_{4}\right)-y_{2} y_{3} e_{3} e_{4} e_{7}\right] / Q} \\
{\left[y_{2}\left(e_{1} e_{2}-e_{3}\right) e_{7}\right] / Q} \\
{\left[y_{3}\left(e_{4} e_{5}-e_{6}\right) e_{7}\right] / Q} \\
{\left[\left(1-y_{1} e_{2} e_{7}\right)\left(e_{5} e_{7}-y_{4} e_{6}\right)-y_{2} y_{3} e_{2} e_{6} e_{7}\right] / Q}
\end{array}\right)
$$

$\operatorname{avec} Q=\left(1-y_{1} e_{2} e_{7}\right)\left(e_{7}-y_{4} e_{4}\right)-y_{2} y_{3} e_{2} e_{4} e_{7}$ et où $x_{i}$ et $y_{i}$ sont respectivement les réponses (paramètres $\mathrm{S}$ bruts) et les stimuli (paramètres $S$ attribués) pour un étalon donné, l'indice $i$ faisant correspondre respectivement les valeurs $1,2,3$ et 4 aux paramètres $S$ d'indices $11,12,21$ et 22 . L'indice $j$ désignant l'étalon est omis par souci de simplicité.

Dans le cas de la ligne, le système d'équations (8) devient

$$
\left(\begin{array}{l}
x_{1} \\
x_{2} \\
x_{3} \\
x_{4}
\end{array}\right)=\left(\begin{array}{c}
\left(e_{1}-T^{2} e_{3} e_{4}\right) /\left(1-T^{2} e_{2} e_{4}\right) \\
T\left(e_{1} e_{2}-e_{3}\right) /\left(1-T^{2} e_{2} e_{4}\right) \\
T\left(e_{4} e_{5}-e_{6}\right) /\left(1-T^{2} e_{2} e_{4}\right) \\
\left(e_{5}-T^{2} e_{2} e_{6}\right) /\left(1-T^{2} e_{2} e_{4}\right)
\end{array}\right)
$$

et on peut noter que les valeurs étalons de la ligne de transmission (correspondant à ses paramètres $S_{11}$ et $S_{22}$, ici $y_{1}$ et $y_{4}$ ) disparaissent bien des équations du modèle.

Après avoir estimé les paramètres d'erreur, en s'appuyant sur l'un des algorithmes TRL déjà publiés [14], on peut en inversant le système d'équations, donner les stimuli estimés correspondant aux réponses observées, en fonction des paramètres d'erreur estimés (fonction $\mathbf{h}$ ) :

$$
\left(\begin{array}{c}
\widehat{y_{1}} \\
\widehat{y_{2}} \\
\widehat{y_{3}}
\end{array}\right)=\left(\begin{array}{c}
{\left[\left(x_{1}-\widehat{e_{1}}\right)\left(x_{4} \widehat{e_{4}}-\widehat{e_{6}}\right)-x_{2} x_{3} \widehat{e_{4}}\right] /\left(\widehat{e_{7}} R\right)} \\
x_{2}\left(\widehat{e_{4}} \widehat{e_{5}}-\widehat{e_{6}}\right) / R \\
x_{3}\left(\widehat{e_{1}} \widehat{e_{2}}-\widehat{e_{3}}\right) / R \\
{\left[\left(x_{1} \widehat{e_{2}}-\widehat{e_{3}}\right)\left(x_{4}-\widehat{e_{5}}\right)-x_{2} x_{3} \widehat{e_{2}}\right] \widehat{e_{7}} / R}
\end{array}\right)
$$

$\operatorname{avec} R=\left(x_{1} \widehat{e_{2}}-\widehat{e_{3}}\right)\left(x_{4} \widehat{e_{4}}-\widehat{e_{6}}\right)-x_{2} x_{3} \widehat{e_{2}} \widehat{e_{4}}$.

Grâce à ce procédé, les incertitudes peuvent être propagées de manière globale, l'incertitude pouvant être attribuée à la fois aux valeurs étalons et à la mesure. Une incertitude est associée aux mesures à cause des imperfections du système, telles que les erreurs de bruit, dues aux câbles et aux connecteurs, etc. Une incertitude est associée aux valeurs étalons car les objets physiques n'ont pas des propriétés idéales, de sorte que les valeurs utilisées dans les équations d'étalonnage sont entachées d'erreurs. Ainsi, les valeurs étalons (stimuli) $y_{i j}$ sont déclarées en nombres incertains de même que les mesures brutes (réponses) correspondantes $x_{i j}$, en attribuant aux unes et aux autres des incertitudes sur les parties réelles et imaginaires.

\section{Exemple : cas d'un atténuateur}

Nous appliquons le procédé présenté au cas de la mesure corrigée d'un atténuateur de $20 \mathrm{~dB}$ en connecteur PC7 en utilisant un kit d'étalonnage également en connecteur PC7, utilisable de $2 \mathrm{GHz}$ à $18 \mathrm{GHz}$.

Après avoir mesuré les trois étalons Thru, Reflect et Line, en appliquant un algorithme TRL, nous déterminons les termes d'erreur sous la forme de nombres incertains auxquels est d'abord associée une incertitude due aux mesures.

Ensuite, en n'utilisant que la valeur sans incertitude des termes d'erreur, nous recalculons par l'équation (8) le résultat de la pseudo-mesure, que l'on obtient sous la forme de nombres incertains contenant l'incertitude due aux étalons.

L'algorithme est alors exécuté une seconde fois pour déterminer les termes d'erreur avec l'incertitude due aux étalons. Il suffit alors de combiner les deux résultats pour obtenir les termes d'erreur avec l'incertitude due à la fois aux étalons et à la mesure.

A ce stade, nous pouvons mesurer un composant donné et calculer via l'équation (10), ses paramètres $\mathrm{S}$ 
corrigés (attribués) et leur incertitude associée. Cette incertitude dépend alors à la fois des incertitudes attribuées aux mesures et aux étalons.

Dans les tableaux 1 et 2 nous indiquons les composantes typiques retenues et leurs valeurs pour traiter cet exemple, respectivement attribuées à la mesure et aux étalons.

Sur les figures 1 et 2 , nous montrons la répartition des composantes d'incertitude respectivement pour les paramètres $S_{11}$ et $S_{21}$ de l'atténuateur. Pour chaque paramètre $S, 90$ composantes d'incertitude sont prises en compte. Sur les figures 1 et 2 seules les 8 dernières composantes sont dues aux étalons.

On peut voir sur la figure 1, que les composantes dues aux étalons qui peuvent être non négligeables sont celles qui correspondent au $S_{11}$ de la ligne (Line), au $S_{11}$ de la connexion directe (Thru) et à l'impédance caractéristique de la ligne (Line), respectivement représentées par les points marqués $\mathrm{A}, \mathrm{B}$, et $\mathrm{C}$. Sur la figure 2, seule la composante due au $S_{21}$ de la connexion directe (Thru), correspondant au point marqué $\mathrm{D}$, peut ne pas être négligée parmi les composantes dues aux étalons.

Tableau 1. Incertitudes attribuées à la mesure.

\begin{tabular}{|c|c|}
\hline composante & valeur \\
\hline câbles & $10^{-3}$ \\
\hline connecteurs & $10^{-3}$ \\
\hline bruit de plancher & $10^{-6}$ \\
\hline bruit de trace & $5 \cdot 10^{-3}$ \\
\hline isolation & $5 \cdot 10^{-5}$ \\
\hline linéarité & $3 \cdot 10^{-3}$ \\
\hline
\end{tabular}

Tableau 2. Incertitudes attribuées aux étalons.

\begin{tabular}{|c|c|}
\hline composante & valeur \\
\hline $\begin{array}{c}\text { paramètres S de la } \\
\text { connexion directe } \\
\text { (Thru) }\end{array}$ & $10^{-4}$ \\
\hline $\begin{array}{c}\text { Paramètres } S_{11} \text { et } S_{22} \\
\text { de la ligne (Line) }\end{array}$ & $8 \cdot 10^{-4}$ \\
\hline $\begin{array}{c}\text { symétrie des charges } \\
\text { (Reflect) }\end{array}$ & $5 \cdot 10^{-4}$ \\
\hline $\begin{array}{c}\text { impédance } \\
\text { caractéristique de la } \\
\text { ligne }\end{array}$ & $4 \cdot 10^{-2} \Omega$ \\
\hline
\end{tabular}

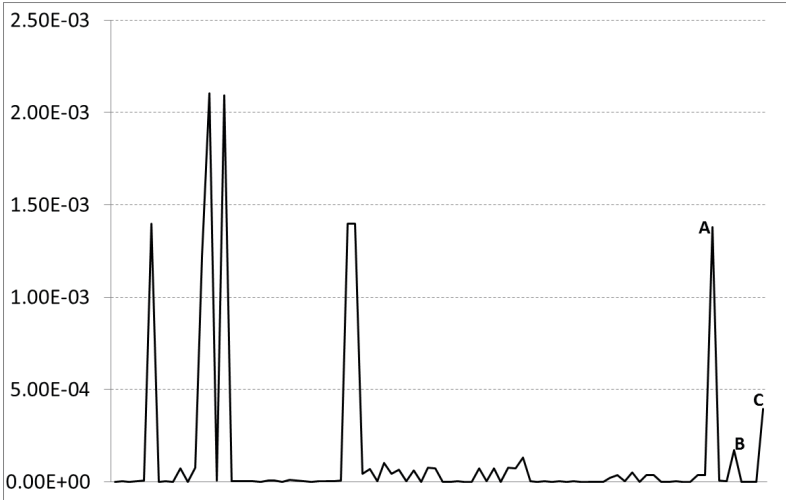

Figure 1. Composantes d'incertitude pour le paramètre $S_{11}$ de l'atténuateur

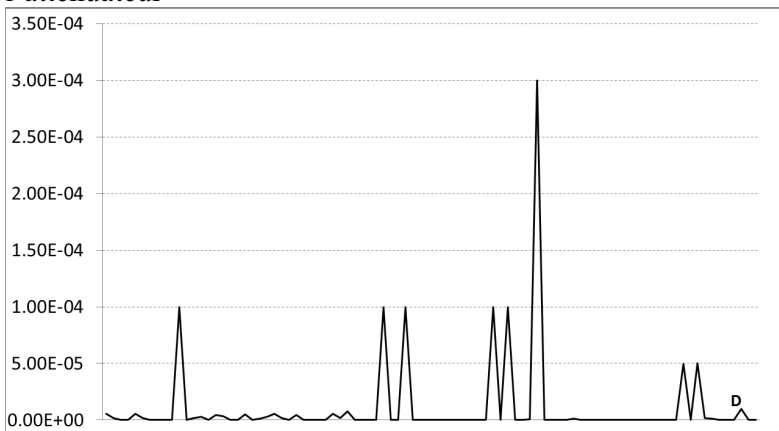

Figure 2. Composantes d'incertitude pour le paramètre $S_{21}$ de l'atténuateur

\section{Conclusion}

Nous avons présenté une méthode permettant de propager les incertitudes dues aux étalons, dans le cas d'une procédure d'étalonnage de type TRL pour laquelle par définition, les équations ne réfèrent qu'aux mesures brutes et pas aux valeurs étalons. Cette propagation se fait de manière globale, associant également les autres composantes d'incertitude, dues à la mesure. Cette méthode est basée sur un calcul indirect des coefficients de sensibilité et peut être automatisée via le concept des nombres incertains.

L'étalon de type ligne de transmission est traçable aux unités du SI par des mesures dimensionnelles. Ainsi, grâce à la méthode décrite dans cet article, il devient possible d'établir la traçabilité des mesures de paramètres $\mathrm{S}$ directement via l'étalonnage, c'est-à-dire sans passer par des étalons de vérification.

\section{Références}

1. G.F. Engen, C.A. Hoer, IEEE MTT, 27(12) (1979)

2. J. Stenarson, K. Yhland, IEEE MTT, 57(11) (2009)

3. U. Stumper, Adv. Radio Sci., 5, (2007)

4. D. Rytting, ARFTG/NIST, San Diego, (2001)

5. BIPM, IEC, IFCC, ISO, IUPAC, IUPAP, OIML, GUM, (2008)

6. BIPM, IEC, IFCC, ISO, IUPAC, IUPAP, OIML, GUM Supplement 2, (2011)

7. B.D. Hall, Metrologia, 43 (2006)

8. B.D. Hall, Meas. Sci. Technol 24 (2013)

9. B.D. Hall, Anamet, Boras, (2013) 